\title{
Experimental Investigation of Real Aperture Synthetically Organised Radar for Breast Cancer Detection
}

\author{
I. J. Craddock ${ }^{(1)}$, R. Nilavalan ${ }^{(1)}$, J. Leendertz $^{(1)}$, A. Preece ${ }^{(1)}$, R. Benjamin ${ }^{(2)}$ \\ (1) University of Bristol, UK \\ (2) 13 Bellhouse Walk, Bristol, UK
}

\section{Introduction}

Breast cancer is the most common cancer in woman, and early detection increases the likelihood of successful treatment and long-term survival. Screen film mammography is currently the most effective method for detecting breast tumours, however this technique suffers from relatively high false negative and positive detection rates, and it involves uncomfortable compression of the breast.

Microwave detection of breast tumours is a potential alternative. It involves no exposure to ionising radiation, and it benefits from the significant dielectric contrast between normal tissue and malignant tumours. A number of groups (e.g. [1]) have therefore proposed microwave-based systems in which, in an analogous fashion to Ground Penetrating Radars, microwaves are transmitted from an antenna or antenna array, and the received signals, which contain reflections from tumours, are recorded and analysed.

The work presented herein originated as a theoretical study employing FDTD models, which produced very promising results [2]. This contribution presents subsequent experimental validation using a mechanically-scanned 2 element antenna array and a breast phantom consisting of synthetic biological materials [3].

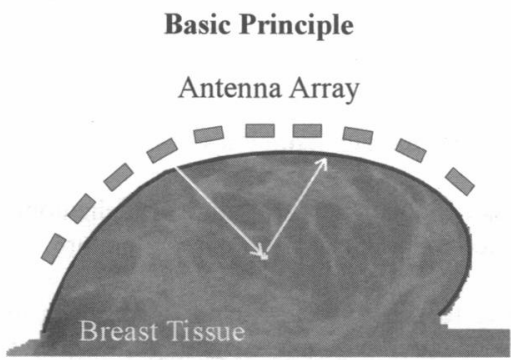

Figure 1: Approximation to envisaged implementation

The work presented here is based on a real aperture post-reception synthetically focussed detection algorithm [2]. In the simplest implementation of the proposed system all elements of an array (see Figure 1) transmit a broadband pulse in turn. 
All other elements sharing any operationally relevant 3D field of view with the current transmit element then record the received signal. By predicting the roundtrip path delay via any desired point in the medium, it is then possible to retrospectively extract and time-align all the signals from that point. This approach is similar in essence to other time-shifting algorithms e.g. [1], except that the radar observations are bistatic in nature rather than monostatic. This offers additional opportunities for processing gain and clutter rejection.

\section{Experimental Evaluation}

A planar antenna design was established with good operational performance over the band $4.5 \mathrm{GHz}$ to $9.5 \mathrm{GHz}$ [4]. Allowing for the refractive index of the medium and the proposed aperture size, this should yield adequate resolution.

Synthetic biological materials for breast tissue, skin and tumour were also developed. In the case of healthy breast tissues, the objective was a relative permittivity close to 10 over the frequency range $4 \mathrm{GHz}$ to $10 \mathrm{GHz}$, together with an attenuation of $4 \mathrm{~dB} / \mathrm{cm}$ at $10 \mathrm{GHz}$ - an emulsion of oil and water was found to have suitable properties (Figure 2).

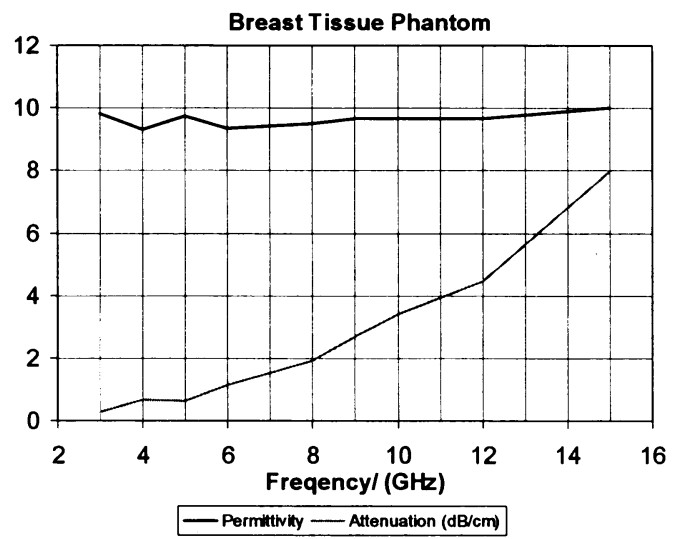

Figure 2: Dielectric properties of the breast tissue medium

Similarly, flat "skin" and spherical "tumour" phantoms were produced using measured dielectric properties taken from the literature.

Before committing to the construction of a complete array of antennas (as in Figure 1) and associated microwave switching network, it was decided to employ merely two independently mechanically-scanned antennas. The two antennas are moved across a horizontal plane by computer-controlled stepper motors. This mechanical movement closely approximates the expected results from a $4 \times 4$ fullypopulated array - at the expense of a dramatically increased measurement time. 
Frequency domain data collection was performed using an Agilent 8722ES Network analyser under GPIB control. This recorded the $S_{21}$ parameter over a frequency band from 4.5 to $9.5 \mathrm{GHz}$ for the 120 unique combinations of transmit and receive antenna positions. The frequency domain data was weighted in order to synthesize a modulated Gaussian pulse and then transformed to the time domain using an Inverse Fast Fourier Transform algorithm.

Figure 3 shows the experimental setup, which includes the pair of antennas, network analyser and the synthetic materials for tumour, skin and breast tissues. These initial experiments were conducted with a $1.5 \mathrm{~mm}$ thick skin phantom.

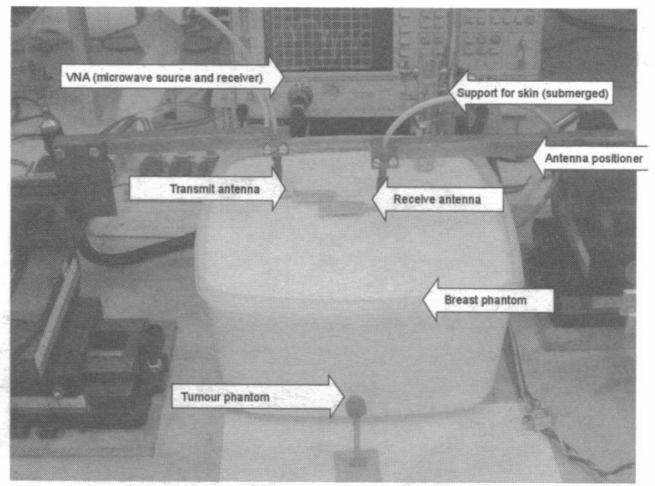

Figure 3: Mechanically-scanned experimental set-up

Typical data is shown in Figure 4, where a $6 \mathrm{~mm}$ tumour is successfully detected. It is important to note that, while there are a few artifacts in this result (arising from the skin reflection), the detection does not involve a background subtraction.

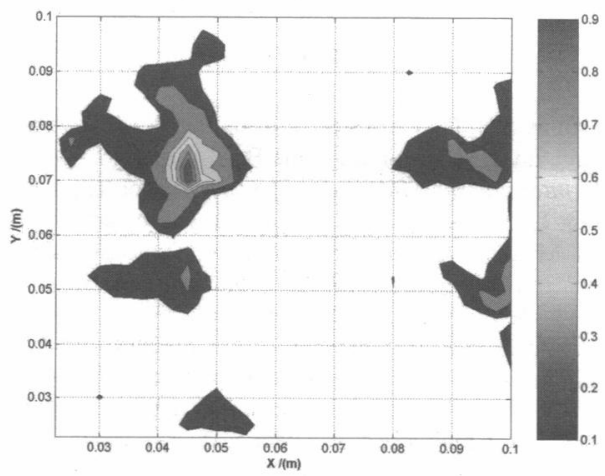

Figure 4: Horizontal slice at tumour location for $6 \mathrm{~mm}$ tumour 


\section{Conclusions}

Initial experimental validation using phantom materials and a mechanicallyscanned pair of antennas has yielded promising results - due to limited space only a single example is shown here. The detection of a $6 \mathrm{~mm}$-diameter phantom tumour in a medium with electrical properties that realistically mimic typical breast tissue, without recourse to background subtraction, is particularly pleasing.

With the confidence in the technique established, the team has recently constructed a fully-populated antenna array (Figure 5) and has now commenced measurements with this new prototype, from which it is hoped to present results at the conference.

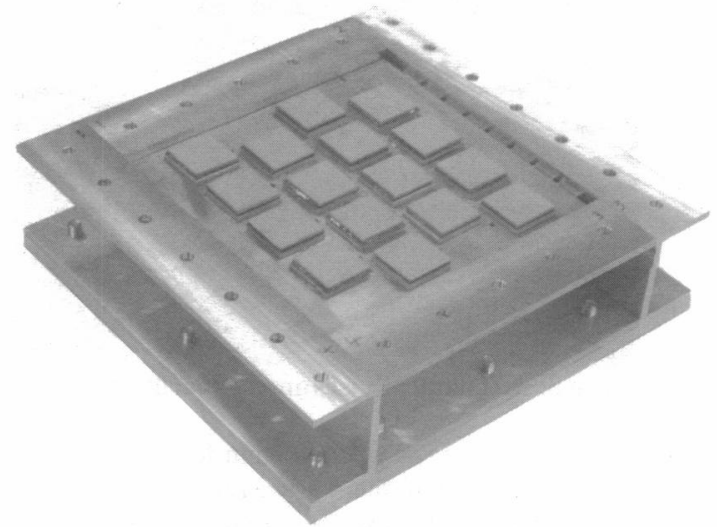

Figure 5: Next-generation array prototype

\section{References:}

[1] X. Li, S. Hagness, B. D. Van Deen, D. van den Weide, "Experimental investigation of Microwave Imaging via Space-Time Beamforming for Breast Cancer Detection," Proc. IEEE International Microwave Symposium, vol. 1, pp 379-382, 2003.

[2] R. Nilavalan, J. Leendertz, I. J. Craddock, A. Preece and R. Benjamin, "Numerical Analysis of Microwave Detection of Breast Tumours using Synthetic Focussing Techniques," in Proc. of the IEEE AP-S International Symposium and USNC/URSI National Radio Science Meeting, Monterey, California, USA, June 2004.

[3] J. Leendertz, A. Preece, R. Nilavalan, I. J. Craddock and R. Benjamin, "A Liquid Phantom Medium for Microwave Breast Imaging," 6th International Congress of the European Bioelectromagnetics Association, Budapest, Hungary, Nov 2003

[4] R. Nilavalan, I. J. Craddock, J. Leendertz, A. Preece, R. Benjamin, "A Wideband Planar Antenna for In-body Imaging," submitted to the IEEE AP-S International Symposium and USNC/URSI National Radio Science Meeting, Washington DC, July 2005 